\title{
Heterotrophic ossification post total knee arthroplasty in a patient with rheumatoid arthritis: a case report
}

\author{
Aswin Thankachan Veliyil*, Dileep S., Renjit John Mathew, John Thayyil John
}

Department of Orthopedics, Lourdes Hospital, Kochi, Kerala, India

Received: 18 September 2021

Accepted: 19 October 2021

\section{*Correspondence:}

Dr. Aswin Thankachan Veliyil,

E-mail: aswinthankachan@gmail.com

Copyright: (c) the author(s), publisher and licensee Medip Academy. This is an open-access article distributed under the terms of the Creative Commons Attribution Non-Commercial License, which permits unrestricted non-commercial use, distribution, and reproduction in any medium, provided the original work is properly cited.

\begin{abstract}
Even though minor degrees of heterotrophic ossification are common in total knee arthroplasty, it is of little clinical significance. But severe degrees of heterotrophic ossification are very rare after total knee arthroplasty. Here we discuss about a 70 years old woman who initially had excellent post-operative range of movements after cemented total knee arthroplasty, but later presented with knee pain, swelling and loss of range of movements after 3 months. X ray showed severe heterotrophic ossification around knee near the quadriceps tendon. She was treated conservatively with nonsteroidal anti inflammatory drugs and physiotherapy. After a period of 3 months of physiotherapy, patient regained the lost range of movements and is currently under follow up for the past 1 year. Hence this case instantiates that even in cases of severe Heterotrophic Ossification after total knee arthroplasty, non-operative treatments such as physiotherapy with anti-inflammatory drugs should be the primary option to treat the stiffness before considering surgery.
\end{abstract}

Keywords: Heterotrophic ossification, Total knee arthroplasty, Physiotherapy

\section{INTRODUCTION}

Stiffness is a calamitous complication after total knee arthroplasty. There are various causes of stiffness after total knee arthroplasty including arthrofibrosis, over sizing of the implants, incorrect gap balancing, heterotrophic ossification etc. Heterotrophic ossification is a condition in which mesenchymal tissues are transformed into osteoblast after tissue trauma. Minor forms of Heterotrophic Ossification are common after knee arthroplasty which is commonly overlooked and are clinically insignificant. Incidence of Heterotrophic Ossification after knee arthroplasty ranges from $4 \%$ to $42 \%$ in literature. ${ }^{1}$ Multiple treatment modalities have been advised for the treatment of heterotrophic ossification after total knee arthroplasty around the knee, including non-operative and operative treatments. Non operative treatment modalities include non-steroidal antiinflammatory drugs, physiotherapy, manipulation under anesthesia and radiotherapy. Operative options include arthroscopic release of adhesions and revision total knee arthroplasty in extreme cases. ${ }^{2}$ In this case report, we discuss about a patient with severe heterotrophic ossification after primary total knee arthroplasty.

\section{CASE REPORT}

A 70-year-old woman presented with right knee osteoarthritis grade 3 (according to Kellgren and Lawrence classification). (Figure 1) She underwent total knee arthroplasty of left knee 6 years back which was uneventful. She had multiple co morbidities like diabetes mellitus, rheumatoid arthritis, hypothyroidism, dyslipidemia for the past 15 years. She was on insulin, thyroxine, methotrexate, hydroxychloroquine and atorvastatin. She weighed $78 \mathrm{~kg}$ and body mass index was 33. Preoperative knee movements were 0 to 90 degree which was painful. There was no history of surgery or 
trauma to left knee in the past. Initial baseline investigations and inflammatory markers were done to rule out any infection. She underwent cemented total knee arthroplasty under spinal anesthesia with tourniquet throughout (Figure 2).

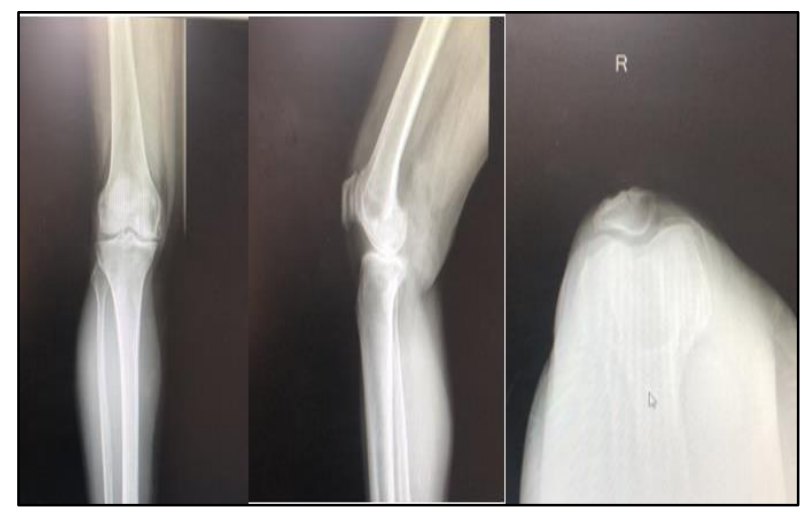

Figure 1: Pre-operative radiographs of right knee.

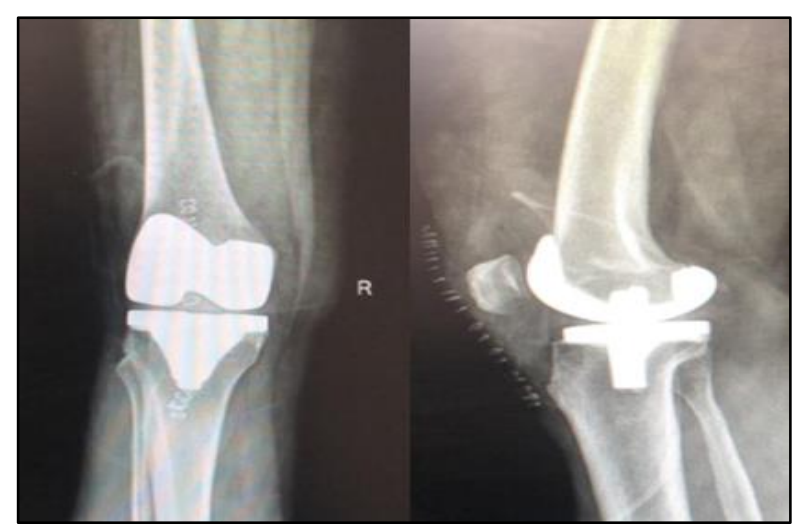

Figure 2: Immediate postoperative radiographs.

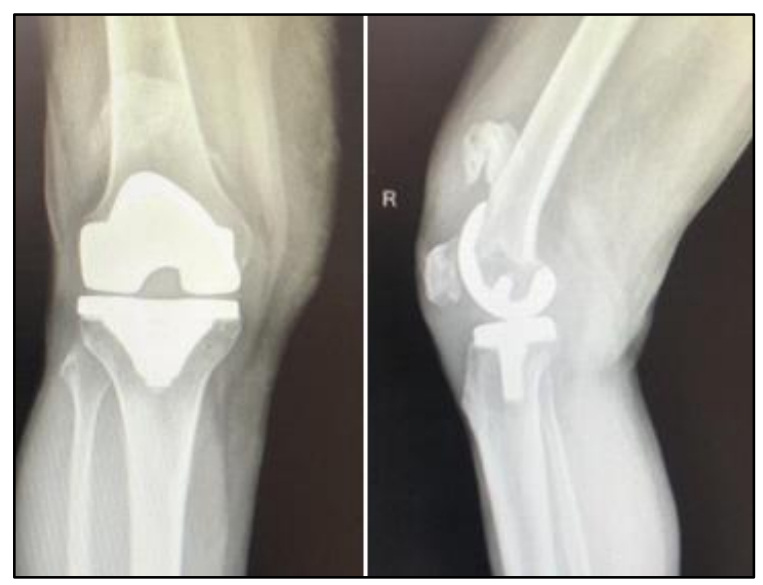

Figure 3: Radiographs at 12 weeks follow-up.

The standard medial parapatellar approach was used and was performed by an arthroplasty surgeon who has more than 20 years' of experience. Intraoperatively, there was no significant soft tissue injury. Minor dissection was performed and complete hemostasis was obtained. Post- operative period was uneventful; drain was minimal, no postoperative knee effusion was there. Full weight bearing mobilization and range of movement exercises were allowed since the first postoperative day. While doing surgery, her bones were found to be very osteoporotic during bone cuts hence was advised teriparatide injection and calcium postoperatively. She was discharged safely on the third postoperative day.

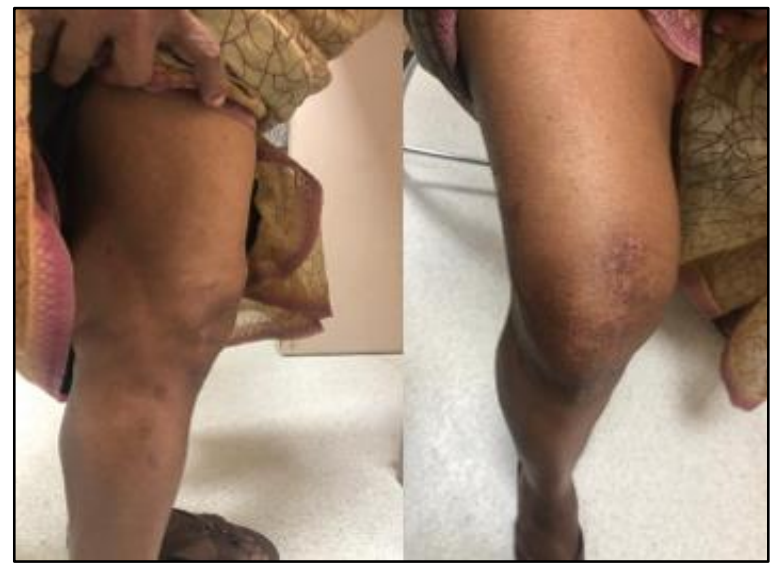

Figure 4: Clinical pictures at 8 months follow up.

The stitches were removed 2 weeks later. At that time range of movement of the right knee was from 0 to 120 degrees. She didn't turn up for follow up at 6 weeks due to lockdown following COVID-19 crisis. She didn't suffer from any trauma to the knee postoperatively and there was history of massage. At 12 weeks, she presented with pain and stiffness of right knee. On examination, she had warm and swollen right knee and her knee movements were restricted. There was flexion deformity of 10 degree with further flexion up to 90 degrees. X ray revealed calcification over quadriceps region suggestive of Heterotrophic Ossification (Figure 3). Blood tests revealed a CRP of 7, ESR of 85, ALP 670 (normal: 80-290 IU/L) and total count were within normal limits. Aspiration of the joint did not reveal any evidence of infection. After a review of the literature, a decision was made to treat the loss of range of motion non-operatively, with non-steroidal anti-inflammatory medication and aggressive physiotherapy. Symptoms gradually improved and by 8 months, she regained a range of motion from 0 degree to 110 degree which is painless. (Figure 4)

\section{DISCUSSION}

Total knee arthroplasty in rheumatoid arthritis patients is shown to have excellent results when compared to osteoarthritis patients in the literature. The pathology of Rheumatoid Arthritis affecting the knee joint makes total knee arthroplasty an attractive option for patients failing medical therapy. Some studies have suggested total knee arthroplasty improves both knee function and disease activity in patients with rheumatoid arthritis. $^{3}$ The incidence of symptomatic Heterotrophic Ossification following total knee arthroplasty is relatively rare. 
Symptoms are present in less than $1 \%$ of patients and common complaints are loss of motion, pain, or quadriceps snapping. Numerous risk factors that have attributed Heterotrophic Ossification are previous history of Heterotrophic Ossification, male sex, ankylosing spondylitis, Paget disease, burns patients, head injury patients and idiopathic skeletal hyperosteosis and previous history of surgery of the affected knee. ${ }^{4}$ Surgical techniques also play an important role in the development of Heterotrophic Ossification. They are excessive periosteal injury after exposure of the distal femur for instrumentation positioning, notching of the anterior cortex, damage to the quadriceps muscle, forced manipulation, inadequate hemostasis, knee hematoma and post-operative knee effusion. ${ }^{5}$ Many studies found that Heterotrophic Ossification formation mainly occurs on the anterior aspect of distal femur, which affects the extensor mechanism, thereby affecting knee flexion. Several classification systems have been proposed for the grading of Heterotrophic Ossification after total knee arthroplasty. None of them include clinical considerations or the need for surgical treatment. There is correlation of size of Heterotrophic Ossification with the overall functional outcome following primary total knee arthroplasty. Dalury et al evaluated 500 patients who underwent cemented Total knee arthroplasty. In their series, $8 \%$ of patients had type $1(<2 \mathrm{~cm})$ new bone formation, $6 \%$ had type $2(2$ to 5 $\mathrm{cm})$ new bone formation, and only $1 \%$ had type $3(>5 \mathrm{~cm})$ new bone formation. The overall incidence of Heterotrophic Ossification was $15 \%$. There was increased incidence of Heterotrophic Ossification in patients who had a preoperative knee deformity of more than 15 degrees, especially in male patients and obese patients. ${ }^{6}$ The efficacy of non-steroidal anti-inflammatory drugs and local radiation therapy for the prevention of Heterotrophic Ossification have been studied and well documented for the hip and can also be applied for the knee. Various studies have recommended Radiotherapy either $4 \mathrm{~h}$ before or within $72 \mathrm{~h}$ of surgery given as a single dose of $8 \mathrm{~Gy}$ or Indomethacin: $75 \mathrm{mg}$ per day for 6 weeks as prophylaxis. Surgical options like open or arthroscopic release of adhesions, removal of Heterotrophic Ossification mass and revision total knee arthroplasty are rarely required. ${ }^{7}$ Hence, the literature on surgical interventions for symptomatic Heterotrophic Ossification after total knee arthroplasty is limited to case reports and in most of the cases surgical excision was used specifically to remove the symptomatic bony masses found in the quadriceps muscle that led to limited range of motion, pain, and external snapping. Alturki et al has reported a case in which severe Heterotrophic Ossification which was not improved by physiotherapy was treated with revision total knee arthroplasty with rotating hinge knee prosthesis. ${ }^{1}$ Surgery is planned only if stiffness due to Heterotrophic Ossification is interfering with his or her daily routines. Surgery if recommended is planned when the Heterotrophic Ossification has matured enough, around 18 months postoperatively and the biochemical markers like CRP and ALP levels are within normal limits. In most of the studies, Heterotrophic Ossification did not have a major impact on the functional outcome of total knee arthroplasty. Even in cases of severe Heterotrophic Ossification, non-operative treatments such as physiotherapy and non-steroidal anti-inflammatory drugs produce acceptable functional results in long term. ${ }^{2}$ There is no general consensus regarding prophylaxis against Heterotrophic Ossification after primary total knee arthroplasty. We did not use prophylaxis against Heterotrophic Ossification prior to total knee arthroplasty in this case. A comprehensive study must be conducted to reach a consensus regarding the prophylaxis using either radiation or Non-steroidal anti-inflammatory drugs, or both, prior to total knee arthroplasty in high-risk patients. According to Dalury et al proposed classification, our case belonged to type 3 heterotrophic ossification. Even in this case we were able to achieve acceptable range of motion with physiotherapy.

\section{CONCLUSION}

The incidence of Heterotrophic Ossification after total knee replacement is probably higher than what is commonly expected, with reported case series incidence of between 4 and $40 \%$. The incidence of severe Heterotrophic Ossification is much less, with only a small number of reported cases. Excessive periosteal injury after exposure of the distal femur for instrumentation positioning, notching of the anterior cortex, damage to the quadriceps muscle, forced manipulation and inadequate hemostasis are the main implicated surgical factors for development of Heterotrophic Ossification. Even in cases of severe Heterotrophic Ossification, non-operative treatments such as physiotherapy and non-steroidal anti-inflammatory drugs produced good long-term results. Surgical options are limited to extreme cases only.

\section{Funding: No funding sources Conflict of interest: None declared Ethical approval: Not required}

\section{REFERENCES}

1. Alturki AA, Aldeghaither SA, Alhandi AA. Severe heterotopic ossification post Total Knee Arthroplasty in a patient with rheumatoid arthritis: a case report. J Surg Case Rep. 2020;2020(3):rjz390.

2. Dodds AL, Keene GCR. Severe Heterotopic Ossification following Total Knee Replacement. Case Rep Orthop. 2014;2014:1-3.

3. Total Knee Arthroplasty improves both knee function and disease activity in patients with rheumatoid arthritis: Modern Rheumatology. Available at: https://www.tandfonline.com/doi/abs /10.1080/14397595.2016.1265705. Accessed on 4 June 2021.

4. Furia R, Pellegrini VD. Heterotopic Ossification Following Primary Total Knee Arthroplasty. 2002;1:7-10. 
5. Baroudi M, Derome P, Malo M. Severe heterotopic ossification and stiffness after revision knee surgery for a periprosthetic fracture.

6. Dalury DF, Jiranek WA. The incidence of heterotopic ossification after Total Knee Arthroplasty. J Arthroplasty. 2004;19(4):447-52.
7. Kachewar S, Sankaye S. Heterotopic ossification following total knee replacement surgery. J Mahatma Gandhi Inst Med Sci. 2013;18(2):122.

Cite this article as: Veliyil AT, Dileep S, Mathew RJ, John JT. Heterotrophic ossification post total knee arthroplasty in a patient with rheumatoid arthritis: a case report. Int J Res Orthop 2021;7:1227-30. 